\title{
Reduction of angiocidin contributes to decreased HepG2 cell proliferation
}

\author{
Guan $\mathrm{XG}^{1}$, Guan $\mathrm{XQ}^{2}$, Feng $\mathrm{K}^{3}$, Jian $\mathrm{R}^{3}$, Tian $\mathrm{D}^{1}$, Tian $\mathrm{D}^{1}$, Tong $\mathrm{HB}^{1}, *$ Sun $\mathrm{X}^{1}$
}

1. Life Science Research Center, Beihua University, Jilin, Jilin Province, China

2. Department of Cardiothoracic surgery, The Second Hospital Affiliated to Lanzhou University, Lanzhou University, Lanzhou, Gansu Province, China

3. College of Chemistry and Biology, Beihua University, Jilin, Jilin Province, China

\begin{abstract}
Background: Angiocidin plays a key role in angiogenesis and tumor progression. High angiocidin expression is detected in some kind of solid tumors and tumor vascular endothelial cells. Several reports have shown the inhibition of angiogenesis and tumor growth caused by angiocidin. However, the role of angiocidin in liver cancers growth is still unclear.

Objectives: To examine angiocidin expression in SMMC-7221 and HepG2 cells and the role of angiocidin in liver cancer cell growth.

Methods: RT-PCR and western blot are used in this study to detect angiocidin expression. SiRNA and MTT experiments are used in exploring the role of angiocidin in tumor cell growth.

Results: Our study showed high angiocidin expression in two kinds of liver cancer cells. Angiocidin protein production in HepG2 cells were reduced significantly by siRNA. When HepG2 cells were transfected with siRNA-angiocidin, these cells showed very low proliferation activity compared with control cells. Our study suggests that reduction of angiocidin may contribute to decreased proliferation activity in liver cancer cells.

Conclusion: Angiocidin is highly expressed in liver cancer cells, and it may play a key role in tumor growth of liver cancers. Keywords: angiocidin, siRNA, cell proliferation

African Health Sciences 2013; 13(3): 560 - 564 http:/ /dx.doi.org/10.4314/ahs.v13i3.5
\end{abstract}

\section{Introduction}

Hepatocellular carcinoma (HCC) is one of the most common cancers in the world. Patients with lowgrade HCC tumors often undergo unnoticed in many years, and they always have a poor prognosis when the tumors develop to high-grade. Many genes are involved in the development and growth of HCC, some may work cooperatively in promoting tumor growth $^{1,2}$. Looking for new genes involved in tumor growth may help to uncover the mechanism in HCC development.

Angiocidin was first cloned in 1993 from lung tumor extracts as a tumor cell receptor for CSVTCG, which is a thrombospondin adhesive domain. The amino acid sequence of angiocidin only has three additional amino acids at positions 769-771 compared with anti-secretory factor (ASF) and S5a ${ }^{3}$. Angiocidin proteins are detected in many kinds of

*Corresponding author:
Xin Sun
Life Science Research Center, Beihua University
No.3999 Huashan Road
Fengman District, Jilin, 130022
Tel: 00186-432-64608350
E-mail: sunxinbh@126.com

tumor tissues such as breast carcinoma, head and neck carcinoma, and colon carcinoma ${ }^{4,5}$. Interestingly, high expression of angiocidin are detected in the tumor epithelium, while very low expression in surrounding normal tissues epitheliums ${ }^{6}$. In the serum of HCC patients, angiocidin protein production elevated accompanied by tumor progression ${ }^{7}$, which suggests that angiocidin may be a new gene target for therapy of tumors. Several reports have shown that angiocidin are involved in invasion and angiogenesis of tumors. In human umbilical vein endothelial (HUVE) cells, cell proliferation and migration decreased remarkably when angiocidin expressions were inhibited. The similar result was got in colon cancer cells. Colon cancer cells expressing low- level angiocidin exhibited very low proliferation rates than high expression cells. In vivo, nude mice injected with low angiocidin expression colon cancer cells develop significantly smaller tumors than the mice injected with normal cells ${ }^{8-12}$. These above results suggest that angiocidin may play a key role in tumor growth. However, the role of angiocidin in tumor growth (especially liver cancer) remains largely unknown. 
In this study, angiocidin expression and its role in liver cancer cells were examined. Both SMMC7221 and HepG2 cells showed high angiocidin protein expression. To indicate the role of angiocidin in liver cancer growth, siRNA-angiocidin were designed and used in inhibiting angiocidin protein production. HepG2 cells transfected with siRNAangiocidin showed slower growth compared with control cells. Our study suggests that reduction of angiocidin contributes to the low proliferation activity of HepG2 cells.

\section{Methods}

\section{Cell culture}

SMMC-7221 and HepG2 cells were grown in Dulbecco's modified Eagle medium (DMEM, Sigma) containing 10\% fetal bovine serum, $100 \mathrm{U} /$ $\mathrm{ml}$ penicillin, $100 \mathrm{ug} / \mathrm{ml}$ streptomycin. Monolayer cells were maintained at $37^{\circ} \mathrm{C}$ and $5 \% \mathrm{CO} 2$. When cells grow nearly $60 \%$ confluency, they are passaged. Lipofectamine 2000 (Invitrogen) was used to introduce the plasmids into cells.

\section{Construction of siRNA target to angiocidin}

Two specific siRNA sequences against angiocidin were designed by siRNA Target Finder (Ambion). The sense sequence of siRNA1 is 5'aacaacgtgggccttatcaca-3', and sense sequence of siRNA2 is 5'-aagatgcgcatcattgccttt-3', which were constructed into pSilencer 4.1 CMV-neo vectors. A nonsilencing siRNA was used as negative control. All the constructs were verified by DNA sequencing.

\section{RNA isolation and RT-PCR}

Total RNA was isolated from cells using the Trizol reagent according to the manufacturer's instructions. The prepared RNA was then reversely transcribed to single-stranded cDNAs using oligo(dT) primers (Invitrogen). The cDNA was as the template in polymerase chain reaction (PCR) using angiocidin specific primers. The angiocidin primers are: 5' aagatggtgttggaaagcac 3', 5' tcacttcttgtcttcctccttc 3'. Beta-actin expression was a negative control in the process of PCR.

\section{MTT assay}

After two days of transfection, HepG2 cells (4000/ well) were seeded onto a 96 -well plate and incubated at $37^{\circ} \mathrm{C}$. Three days later, $20 \mathrm{ul} \mathrm{MTT}(5 \mathrm{mg} / \mathrm{ml}$ in PBS) solution added to each well, and the plate was then incubated at $37^{\circ} \mathrm{C}$ for a further 4 hours. The medium and MTT were removed from the wells and the crystals dissolved in 150ul of DMSO. After 10 min of shaking, the absorbance was measured at $490 \mathrm{~nm}$ (OD490) using a microplate reader.

\section{Immunofluorescence}

Cells were seeded on glass cover slips for $12 \mathrm{~h}$ and then transfected with each indicated constructs. Twenty-four hours after transfection, the cells were fixed with 4\% paraformaldehyde in PBS for $15 \mathrm{~min}$, then washed with PBS and permeabilized with $0.1 \%$ Triton X-100 in PBS for $10 \mathrm{~min}$. The cells were blocked by incubation in PBS containing 2\% BSA (Sigma-Aldrich) for $1 \mathrm{~h}$ at room temperature. The cells were then exposed to a polyclonal rabbit antirat angiocidin antibody $(1: 200)$ overnight at $4^{\circ} \mathrm{C}$. After washing, a Cy3-conjugated sheep anti-rabbit $\mathrm{IgG}$ secondary antibody (1:500, Sigma-Aldrich) was applied for $30 \mathrm{~min}$ at room temperature. The cells were washed with PBS and photographed by an OLYMPUS IX71 microscope.

\section{Western Blot}

After $72 \mathrm{~h}$ of transfection, total protein were extracted with lysis buffer $(150 \mathrm{mM} \mathrm{NaCl}, 20 \mathrm{mM}$ Tris, $5 \mathrm{mM}$ EDTA pH7.5, 1\% Triton X-100, and supplemented with $1 \mathrm{mM}$ PMSF). BCA Protein Assay Reagent kit (Pierce) was used to determine the protein concentration. Equal amounts of protein (10ug) were separated by SDS-PAGE and transferred to PVDF membranes. The membranes were blocked in TBST buffer containing 5\% nonfat milk for $1 \mathrm{~h}$, and incubated with angiocidin antibody (1:500) for $2 \mathrm{~h}$ at room temperature. After three times of washing with TBST buffer, PVDF membranes were then incubated with goat antirabbit IgG horseradish peroxidase conjugated (1:5000) for $1 \mathrm{~h}$ at room temperature. Three times of washing are needed, and incubation with the ECL reagent (Amersham), chemiluminescence signals were detected on X-ray film and quantitated by image analysis.

\section{Results}

\section{Angiocidin expressed in SMMC-7221 and HepG2 cells.}

The angiocidin expression in two kinds of liver cancer cells are examined by RT-PCR and western blot. Fig. 1 a shows that angiocidin proteins are detected in SMMC-7221 and HepG2 cells. There seems to be not significant differences in angiocidin expression between SMMC-7221 and HepG2 cells. Moreover, 
angiocidin mRNA and protein in HepG2 cells are studied through RT-PCR and immunoflurence technology. As can been seen from Figures $1 \mathrm{~b}$ and 1c, we got the conclusion that angiocidin expressed in HepG2 cells.
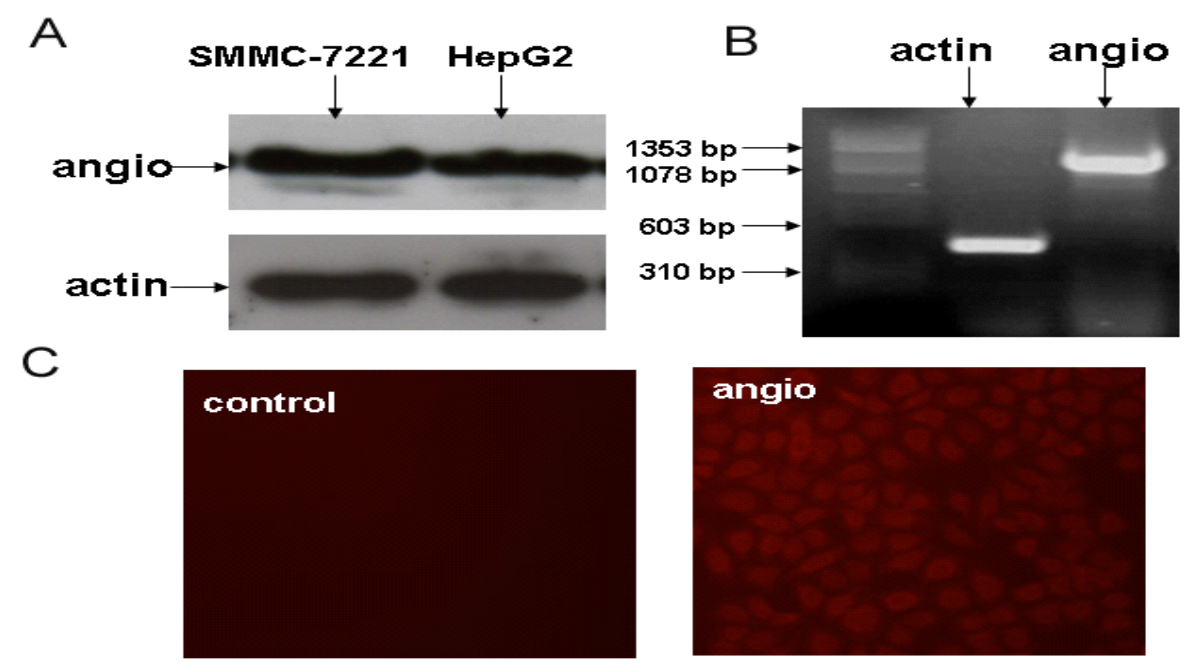

Figure 1: The angiocidin expression in liver cancer cells.

A: Western blot analysis of angiocidin protein expression in SMMC-7221 and HepG2 cells.

B: The angiocidin mRNA expression in HepG2 cell indicated by RT-PCR analysis.

C: Immunofluorescence analysis showing the angiocidin expression in HepG2 cells

siRNA-angiocidin inhibited angiocidin expression in HepG2 cells.

In order to study the role of angiocidin in tumor growth, siRNA- angiocidin were designed and constructed. siRNA-control, siRNA-angio1 and siRNA-angio2 were introduced into HepG2 cells according to the instruction of Lipofectamine 2000. The angiocidin protein production was detected through western blot analysis after $72 \mathrm{~h}$ of transfection. Figure 2 showed that the angiocidin protein productions were reduced by $60 \%$ via
siRNA-angio1, while angiocidin protein in untreated and control cells unchanged. However, angiocidin protein production in cells transfected siRNA-angio2 seems not be influenced compared with control. The unchanged Beta-actin expression in all groups suggested that angiocidin expression was specifically inhibited by siRNA. Our results demonstrated that siRNA-angio1 can remarkably inhibit angiocidin expression in HepG2 cells while siRNA-angio2 does not work in gene silencing.

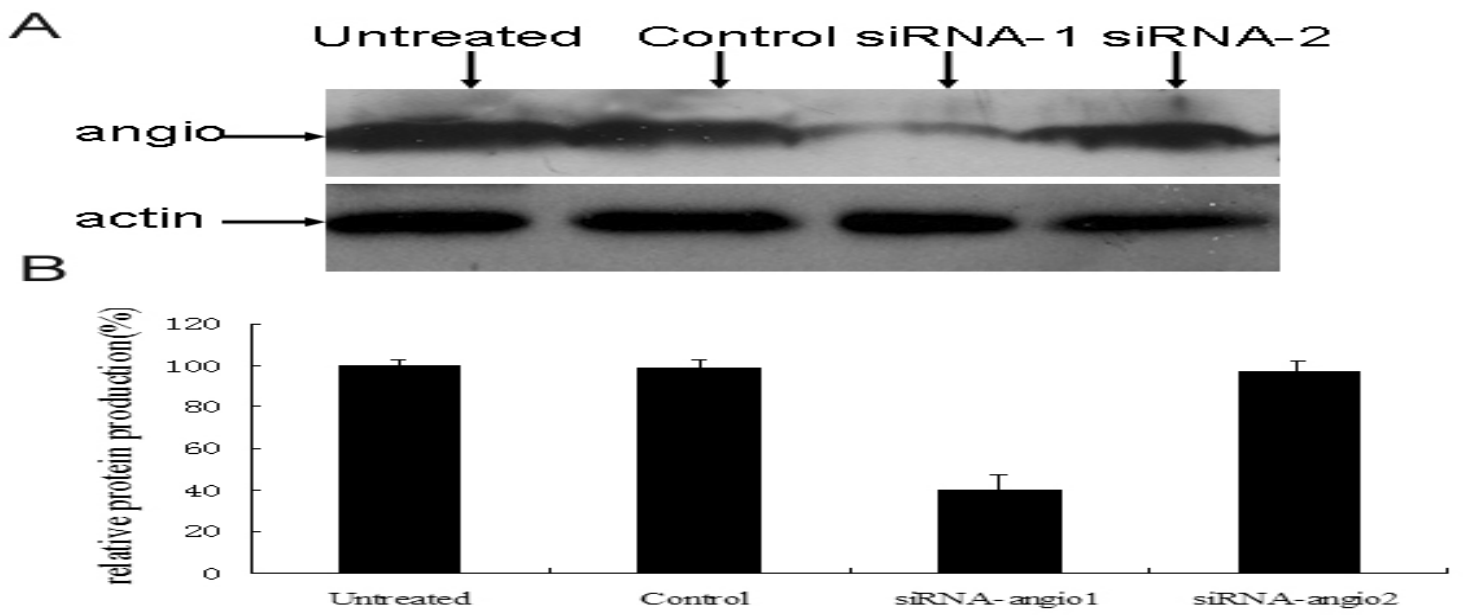

Figure 2: Angiocidin expression was reduced by siRNA-angio in HepG2 cells.

(A) Western blot analysis showing the angiocidin reduction in HepG2 cells.

(B) Quantification of angiocidin protein reduction as in A. Error bars represent standard deviation 
Reduction of angiocidin inhibited the HepG2 cells proliferation.

To examine the role of angiocidin in tumor cell growth, MTT assay was performed using the siRNA-angio1 treated HepG2 cells. Figure 3 showed that cell proliferation in transfected siRNA-angio1 HepG2 cells is much lower than that untreated cells. And siRNA-control has no effect on cell proliferation. These results mean that reduced angiocidin may be associated with slow growth of HepG2 cells.

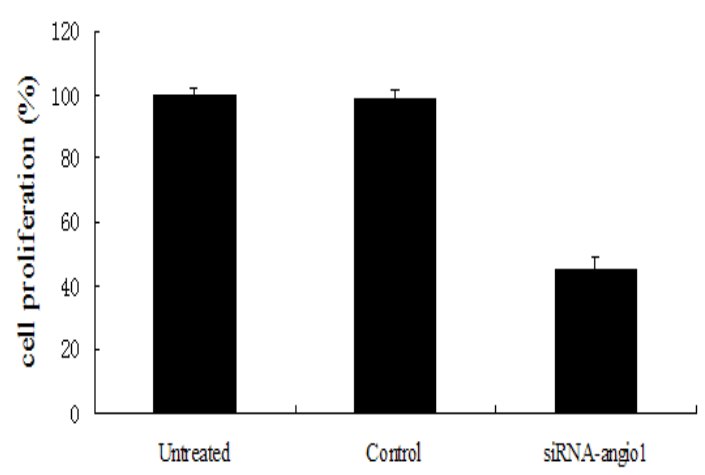

Figure 3: siRNA-angio inhibits cell proliferation activity of HepG2 cells. Error bars represent standard deviation

\section{Discussion}

Accumulating evidence have shown angiocidin expressed in many solid and tumors tumor cell lines such as breast carcinoma, head and neck carcinoma, and colon carcinoma. Over-expressed angiocidin detected in sarcomas and human colon cancer specimens suggests angiocidin expression has a positive correlation with tumor grade. The similar phenomenon was found in hepatocellular carcinoma patients with high angiocidin expression, in contrast to the nearly undetectable levels in healthy persons? Our study also showed that in vitro high angiocidin expressions were detected in two kinds of liver cancer cells, SMMC-7221 and HepG2 cells. These results suggested that angiocidin may contribute to the tumor growth, and it may be a potential gene target for tumor therapy.

Inhibiting angiocidin expression in colon cancer cells and in breast cancer cells have been reported by several groups recently ${ }^{13,14}$. In this study, we indicated for the first time the angiocidin expression in liver cancer cells. Using siRNA, endogenously expressed angiocidin was inhibited significantly in HepG2 cells. The angiocidin protein productions were inhibited significantly via siRNA-angio1.
However, siRNA-angio2 does not work in inhibiting angiocidin production. The failure of siRNA-angio 2 in angiocidin inhibiting may be attributed to off-target effects or other reasons. Our study showed that reduced angiocidin protein may be involved in low cell proliferation of HepG2 cells, suggesting a key role of angiocidin in liver cancer cell growth.

Recently, advances have been made in study role of angiocidin in tumor growth. Godek $\mathrm{J}$ and his colleagues revealed a possible pathway how angiocidin works in tumor cell growth. He suggested by activating epidermal growth factor receptor and nuclear factor kappa (NF-8B), angiocidin can inhibit cell growth in breast cancer cells ${ }^{15}$. Yang $\mathrm{X}$ et al reported that under the regulation of angiocidin matrix metalloproteinase 2 (MMP-2) and gelatinolytic activity contribute to the invasive activity of HUVE cells. It is therefore likely that the study of angiocidin in tumor growth will be an area of intensive investigation in the near future. Further studies are needed to uncover the mechanism.

\section{Conclusion}

In this study, we showed that angicidin protein express highly in two kinds of liver cancer cells, and reduction of angiocidin may contribute to decreased proliferation ability of liver cancer cells.

\section{Acknowledgements}

This research was financially supported by national natural science fund (No. 81150015), Jilin Provincial Scientific and Technologic Development Project (No.20111809), and the Project of Technology Department of Jilin city (No. 201233126). The authors declare that there is no conflict of interests.

\section{References}

1. Varela M, Sala M, Llovet JM, Bruix J. Treatment of hepatocellular carcinoma: is there an optimal strategy? Cancer Treat Rev. 2003; 29(2):99-104.

2. Sia D, Villanueva A. Signaling pathways in hepatocellular carcinoma. Oncology. 2011; 81 (Suppl 1):18-23.

3. Tuszynski GP, Rothman VL, Papale M, Hamilton BK, Eyal J. Identification and characterization of a tumor cell receptor for CSVTCG, a thrombospondin adhesive domain. J Cell Biol. 1993; 120(2):513-521.

4. Zhou J, Rothman VL, Sargiannidou I, Dimitrov S, Qiu C, Smith E, Sheffield J, Sharma M, Tuszynski GP. Cloning and characterization of angiocidin, a tumor cell binding protein for 
thrombospondin-1. J Cell Biochem. 2004; 92(1):125-146.

5. Tuszynski GP, Nicosia RF. Localization of thrombospondin and its

6. cysteine-serine-valine-threonine-cysteineglycine-specific receptor in human breast carcinoma. Lab Invest. 1994; 70(2):228-233.

7. Roth JJ, Reiver DM, Granick MS, Rothman VL, Nicosia RF, Tuszynski GP. Histopathology and clinical assessment correlate with the cysteineserine-valine- threonine-cysteine-glycine (CSVTCG) receptor of thrombospondin-1 in breast tumors. Histol Histopathol. 1997; 12(4):10131018.

8. Sabherwal Y, Rothman VL, Poon RT, Tuszynski GP. Clinical significance of serum angiocidin levels in hepatocellular carcinoma. Cancer Lett. 2007; 251(1):28-35.

9. Sabherwal Y, Rothman VL, Dimitrov S, L'Heureux DZ, Marcinkiewicz C, Sharma M, Tuszynski GP. Integrin alpha2beta1 mediates the anti-angiogenic and anti-tumor activities of angiocidin, a novel tumor-associated protein. Exp Cell Res. 2006; 312(13): 2443-2453.

10. Sabherwal Y, Rothman V, Albo D, Tuszynski G. Angiocidin, an endogenous inhibitor of angiogenesis, is increased in the sera of cancer patients. Proc Am Assoc Cancer Res. 2004; 45: 1143.
11.Liebig C, Agarwal N, Ayala GE, Verstovsek G, Tuszynski GP, Albo D. Angiocidin inhibitory peptides decrease tumor burden in a murine colon cancer model. J Surg Res. 2007; 142(2): 320-326.

12. Liebig C, Wilks JA, Feig BW, Wang TN, Wilson M, Herdman AV, Albo D. The role of angiocidin in sarcomas. Cancer. 2009; 115(22):5251-5262.

13. Yang X, Rothman VL, L'Heureux DZ, Tuszynski G. Reduction of angiocidin expression in human umbilical vein endothelial cells via siRNA silencing inhibits angiogenesis. Exp Mol Pathol. 2006; 81(2):108-114.

14. L'Heureux DZ, Rothman VL, Tuszynski GP. The interaction of angiocidin with tissue transglutaminase. Exp Mol Pathol. 2010; 88(1):1525.

15. Gaurnier-Hausser A, Tuszynski GP. The immunomodulatory role of angiocidin, a novel angiogenesis inhibitor. Curr Pharm Des. 2009; 15(17):1937-1948.

16. Godek J, Sargiannidou I, Patel S, Hurd L, Rothman VL, Tuszynski GP. Angiocidin inhibits breast cancer proliferation through activation of epidermal growth factor receptor and nuclear factor kappa (NF-8B). Exp Mol Pathol. 2011; 90(3): 244-251. 